\title{
Development, implementation, and assessment of a guided-inquiry teaching-learning sequence on vector calculus in electrodynamics
}

\author{
Laurens Bollen, ${ }^{1, *}$ Paul van Kampen, ${ }^{2, \dagger}$ and Mieke De Cock ${ }^{1, \ddagger}$ \\ ${ }^{1}$ KU Leuven, Department of Physics and Astronomy \& LESEC, \\ Celestijnenlaan 200c, 3001 Leuven, Belgium \\ ${ }^{2}$ Centre for the Advancement of STEM Teaching and Learning \& School of Physical Sciences, \\ Dublin City University, Glasnevin, Dublin 9, Ireland
}

(Received 15 February 2018; published 9 November 2018)

\begin{abstract}
We have developed, implemented, and assessed a teaching-learning sequence that aims to enhance students' understanding of the divergence and curl of electromagnetic fields. We designed guided-inquiry worksheets based on student difficulties we identified during semiquantitative and qualitative studies and discussions in the literature. A multiple representation approach was adopted to strengthen the link between graphical representations of vector fields, calculations involving divergence and curl, and Maxwell's equations in differential form. We used the Design, Functions, Tasks framework to optimize learning with the multiple external representations exhibited in our learning materials. These guided-inquiry worksheets used in small-group tutorials comprise short open-ended questions that encourage discussions in small groups of students. They were implemented in three consecutive years in a second-year electrodynamics course at KU Leuven. The intervention was assessed using the same pretest and posttest design adopted to evaluate learning in the original instruction. In addition, we gauged our students' opinions on the intervention. We observed that our intervention positively affected our students' structural understanding of the vector operators, their ability to interpret divergence and curl in graphical representations of vector fields, and their conceptual understanding of Maxwell's equations in differential form. In addition, our students indicated that they enjoyed the teaching approach, felt they learned something from the worksheets, agreed with the difficulty level of the materials, and would like similar tutorials on different subjects.
\end{abstract}

DOI: 10.1103/PhysRevPhysEducRes.14.020115

\section{INTRODUCTION}

Five mathematical expressions form the foundations of the theory of electromagnetic phenomena: the Lorentz force law and Maxwell's equations. The latter comprise four fundamental laws, which in differential form read as follows:

$$
\begin{array}{ll}
\boldsymbol{\nabla} \cdot \mathbf{E}=\frac{\rho}{\epsilon_{0}} & \text { Gauss's law } \\
\boldsymbol{\nabla} \cdot \mathbf{B}=0 & \text { nameless law } \\
\boldsymbol{\nabla} \times \mathbf{E}=-\frac{\partial \mathbf{B}}{\partial t} & \text { Faraday's law } \\
\boldsymbol{\nabla} \times \mathbf{B}=\mu_{0} \mathbf{J}+\mu_{0} \epsilon_{0} \frac{\partial \mathbf{E}}{\partial t} & \text { Maxwell-Ampère's law }
\end{array}
$$

*Laurens.Bollen@kuleuven.be

Paul.van.Kampen@dcu.ie

Mieke.DeCock@kuleuven.be

Published by the American Physical Society under the terms of the Creative Commons Attribution 4.0 International license. Further distribution of this work must maintain attribution to the author(s) and the published article's title, journal citation, and DOI.
In introductory physics courses, Maxwell's equations are typically formulated in terms of line and surface integrals. In intermediate and advanced courses the differential form is often more convenient. Writing the four laws in terms of divergence and curl not only increases the aesthetic beauty of the mathematical expressions, but also allows, e.g., straightforward derivation of the electromagnetic wave equations. In addition, Maxwell's equations in differential form are the starting point for gauge theory and Einstein's theory of special relativity, both historically and in many physics curricula. However, a correct interpretation of these equations is a challenging task, since it requires the use of advanced mathematical knowledge in a physics context.

The use of mathematics in physics is an important topic in physics education research [1-8]. Various studies report that students struggle to incorporate their mathematical knowledge into physics, because they focus on equations and calculations rather than on the underlying concepts [9-11]. As a result students generally develop a decent operational understanding, since they are acquainted with the mathematical processes, algorithms, and actions, but lack structural understanding of the meaning of the 


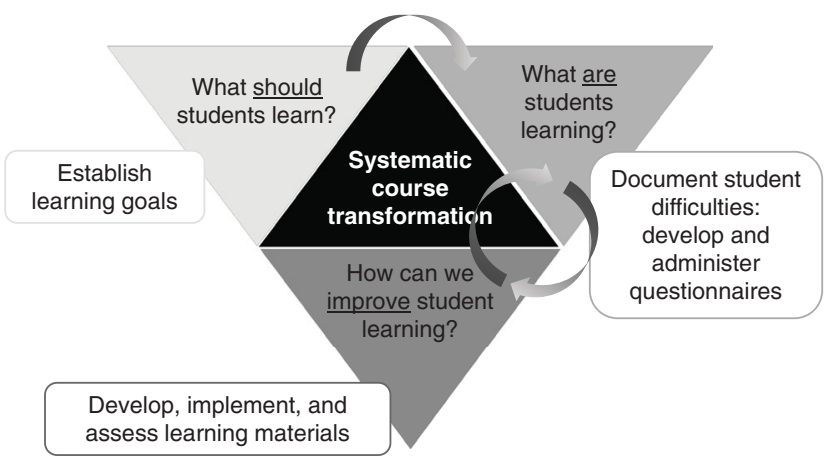

FIG. 1. A model of research-based course transformation. Adapted from Ref. [14].

mathematical entities used in physics [12,13]. For example, students who see divergence and curl merely as the process of adding or subtracting partial derivatives of a vector function have an operational understanding of the concepts; students who see divergence as an object representing the source of a volume flux density, or curl as an infinitesimal rotation, that can be compared at various points and represented in various ways, have a structural understanding.

To enhance students' understanding of the use of vector calculus in electrodynamics, we have adopted the researchbased course transformation procedure patterned after Chasteen et al. [14] shown in Fig. 1. In answer to the question "What should students learn?", we established a set of learning goals based on results from earlier studies, teaching and learning experiences, course textbooks, and aims of electrodynamics modules. We distinguish four skills or competencies that our students need to acquire regarding the use of vector calculus in electrodynamics: (i) structural understanding $[12,13]$ of the vector operators, (ii) calculation of divergence and curl, (iii) graphical interpretation of vector fields in terms of divergence and curl, and (iv) conceptual understanding of Maxwell's equations in differential form.

In order to formulate an answer to the question "What are students learning?", we conducted multiple qualitative and semiquantitative studies. Based on the list of learning goals, we developed and administered written questionnaires to probe students' use of the vector operators in mathematics and electromagnetism contexts. The pretests and post-tests were given before and after instruction, and were completed individually without recourse to textbooks or other resources. This pretest and post-test design allowed us to evaluate students' knowledge before and after the original instruction at KU Leuven and St Andrews, and document student difficulties with divergence and curl in mathematical and electromagnetic contexts at KU Leuven, St Andrews, and Dublin City University (DCU) $[15,16]$. By conducting eight semistructured student interviews, we gained a better understanding of our students' reasoning processes and were able to identify the elements that cue correct application of the vector operators and Maxwell's equations in differential form [17]. A follow-up study probed students' abilities to interpret, construct, and switch between different representations of vector fields [18].

In this article, we intend to answer the question "How can we improve student learning?" The research aims of this study may be summarized as follows:

(1) develop a research-based teaching-learning sequence on divergence and curl in mathematics and physics contexts that uses a multiple representation approach;

(2) implement the materials developed in small-group tutorials that engage students and evoke student discussions in the electrodynamics course;

(3) assess the intervention in both a semiquantitative and qualitative way:

(a) evaluate the effectiveness of the intervention by comparing post-test results with pretest results and findings after the original instruction;

(b) determine student opinions on the tutorials.

In Sec. II, we discuss how we developed guided-inquiry worksheets based on the student difficulties with vector calculus in mathematics and physics identified in the literature and in our own studies. The implementation of the tutorials at KU Leuven is described in Sec. III. In Sec. IV the effectiveness of the intervention and results from informal evaluation by the participating students are presented. In Sec. V we summarize the most important conclusions, discuss the significance of these results, and discuss implications for instruction.

\section{DEVELOPMENT OF RESEARCH-BASED LEARNING MATERIALS}

In this section we discuss the empirical and theoretical foundations we used to develop learning materials that help students improve their understanding of divergence and curl in electrodynamics. In Sec. II A, results regarding student difficulties with vector calculus in mathematics and physics from literature and our own studies are discussed in detail. In Sec. II B, we use the DeFT framework [19-21] to explain aspects that optimize learning with multiple representations. The key content selection is discussed in Sec. II C. The main principles on which the research-based worksheets were designed are exemplified in Sec. II D.

\section{A. Summary of results from investigations into student difficulties}

As the learning materials are based on the student difficulties identified in our earlier studies [15-18] and in the literature, we first describe the most important findings regarding students' understanding of vector fields and vector calculus in mathematical and electromagnetic contexts. We discuss student difficulties with vector field representations (Sec. II A 1), their structural understanding of the vector operators (Sec. II A 2), their ability to perform 
calculations with divergence and curl (Sec. II A 3), their skillfulness to interpret graphical representations of vector fields in terms of divergence and curl (Sec. II A 4), and their conceptual understanding of Maxwell's equations in differential form (Sec. II A 5).

\section{Difficulties with vector field representations}

A vector field may be expressed symbolically, or visualized as a field line diagram or field vector plot. By conducting a research study at four European universities [18], we established an overview of the difficulties students encounter when switching between algebraic and graphical representations of vector fields. Gire and Price [22,23] also reported on a variety of errors students make when sketching graphical representations of vector fields, and argued that instructors should be aware that some representational features may have two potential meanings (e.g., length meaning both distance between points and strength of a field) or do not directly correspond to what is being represented (e.g., smaller spacing of field line diagrams corresponds to a greater magnitude of the field). Likewise Fredlund et al. [24] deemed it important that instructors take time to explain the intended meaning of all the features of a representation. We share the view that students may benefit from instruction that focuses on teaching students how to construct, interpret, and switch between symbolic expressions of vector fields, field line diagrams, and field vector plots.

\section{Difficulties with the conceptual meaning of the vector operators}

A limited amount of research deals with students' understanding of vector operators. Manogue and Dray [25] pointed out that in mathematics courses gradient, divergence, and curl are used in a general and abstract way, while in physics courses they are mostly used in certain symmetries (Cartesian, cylindrical, or spherical). They called this the "vector calculus gap," and argued that it is important to eliminate the gap as such differences in teaching approaches between mathematics and physics courses confuse students.

We have conducted studies $[15,16]$ at KU Leuven, St Andrews, and DCU in which we probed second-year physics and mathematics students' concept images of divergence and curl. The concept image is defined as a unique collection of all the mental processes activated when a person encounters a certain concept [26]. An open-ended pretest question asked students to "interpret (i.e., write down everything you think of when you see) the following operations: $\boldsymbol{\nabla} \cdot \mathbf{A}$ and $\boldsymbol{\nabla} \times \mathbf{A}$." When answering this question, about half of our students wrote a symbolic expression for divergence and curl, but fewer than $20 \%$ gave a conceptual explanation. In addition, students' descriptions of what divergence and curl represent were mostly inaccurate or incorrect. In individual interviews [17], these findings were confirmed: many students described divergence as "a measure for the spreading of the field" and curl as "a measure of bending or rotation of the field." Such descriptions resemble nonscientific dictionary definitions of both words rather than the mathematical concepts. In a refined version of the pre- and post-test, correct and incorrect statements about divergence and curl were gathered and students were asked to indicate with which statements they agreed. We found that difficulties with the concepts of divergence and curl were common in various educational contexts. Students mostly relied on operational understanding and showed little or no structural understanding of the vector operators. To enhance students' structural understanding, an intervention should clarify the meaning of divergence and curl.

\section{Difficulties with performing calculations using divergence and curl}

About $60 \%$ of the KU Leuven students could calculate the divergence or curl of a given vector field on the pretest, which is a high percentage compared to the success rate on questions related to the other skills and competencies. This shows students had been well trained in mathematical techniques in mathematics courses. Since their performance in the post-test was even better, we decided that no further intervention was needed regarding the ability to perform calculations with divergence and curl.

\section{Difficulties with interpreting divergence and curl in graphical representations}

In Sec. II A 1 we discussed students' difficulties with vector field representations. Singh and Maries [27] discussed how graduate students struggle with the interpretation of divergence and curl in field vector plots. They argued that course materials often focus strongly on solving mathematically challenging problems rather than on clarifying the underlying concepts. Similar findings were reported by Ambrose [28], when he asked students to decide whether curl was zero or nonzero in several field vector plots. These findings were confirmed and extended in our studies, in which we asked our students to discuss where the divergence or curl vanished in certain field vector plots. [15] Both before and after instruction, less than half of them gave a correct response. Students were often inconsistent in their problem-solving strategies and tended to rely on their intuitive ideas about divergence and curl that often contradicted mathematical definitions. During the interviews it became clear that many students tackle problems that involve vector fields by initially making a field line diagram or field vector plot of the situation [17]. Since sketching a situation is an important heuristic in physics problem solving, we decided that our learning materials would focus on how to switch between various graphical and symbolical representations of vector fields 
and how to interpret divergence and curl in field vector plots and field line diagrams.

\section{Difficulties with interpreting Maxwell's equations in differential form}

When using divergence and curl in an electromagnetism context, it is appropriate to apply Maxwell's equations in differential form. However, it is also possible to visualize the field or set up an algebraic expression of the vector field and then determine the divergence or curl. A research project at the University of Colorado [29] included a question where students are asked to determine where the divergence of an electric field vanishes for a thin, nonconductive uniformly charged spherical shell. This type of question can be solved with the differential form of Gauss's law in a straightforward way. However, the authors reported that only $26 \%$ of their students gave a correct answer. This was in stark contrast to their calculational success, as students scored $90 \%$ on average when asked to compute the divergence and curl of a vector field given in Cartesian coordinates. Baily et al. [30,31] also showed that students in an intermediate electromagnetism course struggle with the concept divergence, and added that learning about divergence in the context of Gauss's law does not necessarily translate to students' understanding in other contexts like the continuity equation.

These results are in line with our findings from analyzing written questionnaires [15] and conducting individual student interviews [17]. In both studies, we asked students to interpret situations involving electromagnetic fields in terms of divergence and curl. This type of question can be solved using Maxwell's equations in differential form. However, as stated before, many students initially made a sketch of the situation. Both the semiquantitative and qualitative study showed that students struggled to apply all four laws correctly and lacked conceptual understanding of Maxwell's equations. Their difficulties were often related to the local character of the laws. For example, many students stated that the divergence is nonzero everywhere in space when there is a charge distribution somewhere in space. The final goal of our intervention is therefore to improve students' understanding of Maxwell's equations in differential form and enhance their ability to apply those laws in situations involving electromagnetic fields.

One of the most interesting findings of the qualitative part of our study was that many students gave different responses depending on the approach they used: a calculation, the interpretation of a graphical representation, or applying Maxwell's equations. Moreover, the interviewees were unsure which result was correct and struggled to argue how all three approaches could lead to the same response. As a result, our learning materials strongly rely on using multiple representations and aim to clarify the link between the different problem-solving strategies. In the next sections, we discuss in detail on which elements we focus in the tutorials and give some excerpts from the worksheets.

\section{B. A multiple representation approach: The DeFT framework}

The information presented in the previous section suggests that our students experienced difficulties with linking calculations, field vector plots, field line diagrams, and Maxwell's equations in differential form. In addition, they struggled to use multiple symbolic and graphical representations in electrodynamics. However, being able to interpret various representations and being able to switch between formats of concepts and problems are important skills that correlate with a deep understanding of the topic $[32,33]$. Others have reported that learners similarly did not exploit the advantages related to the use of multiple representations in contexts different from ours [34-36], or struggled to switch between different representations. $[18,33,37]$ Since using multiple representations is generally accepted to be an effective instructional approach when aiming to improve students' understanding of a certain concept or operation, [38-46] the materials we developed strongly focus on students learning to link calculations, graphical representations, and Maxwell's equations.

In literature about multiple representations, one often distinguishes between internal and external representations. Zhang and Norman $[47,48]$ explained that internal representations are mental meanings that have to be retrieved from memory, like the numerical value of the symbol 7 . External representations can be perceptually inspected from the environment, like spatial relations between symbols. Internal and external representations can be transformed into each other by externalization and internalization, respectively. Zhang and Norman pointed out that it is important to be aware of the differences and dynamics between internal and external representations, even though most studies only consider one of them.

The majority of research in physics education concerns external representations. Ainsworth [34,36] discussed the importance of using multiple external representations (MER), and described how they can support learning and problem solving. In other papers, Ainsworth and colleagues [19-21] developed and discussed the DeFT framework, which can be used to characterize multirepresentational learning environments. The Design, Functions, Tasks (DeFT) framework sets out a number of questions that designers and teachers should ask in order to provide effective learning experiences for their students.

To benefit from MERs, students should understand how every representation presents information, how different representations relate to each other, and how the most appropriate representation would be chosen in a specific context. Three functions of MERs may be distinguished: to complement, constrain, and construct [34-36]. MERs can be used in complementary roles, where each of the 
representations may contribute to the information or process, like when velocity is represented by both an equation and a $v, t$ graph. The second function of MERs is to improve students' understanding of a domain by using one representation that constrains their interpretation of a second representation. For example, when students make the common error of thinking that an object is at rest when the $v, t$ graph is a horizontal line, a teacher might employ the constraining, more familiar representation of enacting motion at constant speed while showing a real-time graph to support the students' interpretation of the less familiar graphical representation. Finally, MERs can help students to develop a structural understanding ("reification" in the parlance of Ainsworth), which is the function that is most appropriate for our project.

The various possible functions of MERs leave many ways to design multiple representation approaches that vary in terms of content, target users, and teaching strategies. While the choice of approach may influence learning outcomes, Ainsworth and colleagues [19-21] argued that there is a set of five design parameters that uniquely applies to MER approaches: (a) the number of representations, (b) the form of each representation, (c) the way information is distributed, (d) the sequence of representations, and (e) the support for switching between representations.

Taking into account the various functions and design parameters, the DeFT framework then arrives at the development of cognitive tasks. To successfully learn with MERs, the student should understand (i) how every representation presents information, (ii) the relation between the different representations, and (iii) how to choose the most appropriate representation in a specific context.

\section{Design of the materials: Content selection}

The learning materials in the teaching-learning sequence we designed are supplementary to Griffiths's textbook [49] and use multiple graphical and symbolic representations. Many researchers argue that instruction on vector calculus should use methods that facilitate linking symbolic expressions and visualizations [30,31,50-54]; our studies $[15,17,18]$ confirm these statements. By adopting a MERs approach within the DeFT framework we aimed to improve students' abilities to interpret, construct, and switch between different representations of vector fields, and help them make sense of the characteristics of divergence and curl in visualizations of vector fields. As our studies revealed that vector fields with spherical and cylindrical symmetry are very challenging for students, and such fields are very common in physics, the worksheets address such fields repeatedly.

In individual student interviews [17], we helped students gain insight into the divergence and curl of electric and magnetic fields. When answering questions on these topics students would often spontaneously draw field lines and use this representation in their reasoning. However, more often than not this approach led students to erroneous conclusions: they associated diverging field lines with nonzero divergence of the electric field, and bending field lines with nonzero curl of a magnetic field. Most students were able to revise their reasoning after they had been prompted to use symbolic representations and had been given a vector field plot representation. We illustrate in Sec. II D how these findings influenced the design of two worksheets.

There are various teaching approaches that could be used when intervening in a physics course (examples can be found in Ref. [55]). We opted for an approach based on guided-inquiry worksheets, each consisting of a set of structured questions that guide students to reconstruct their concept images, learn various solution strategies, learn to switch between different representations, and link mathematical entities to physical phenomena. Many studies [56-66] have confirmed that such an actively engaging approach is generally more effective than traditional instruction.

Using the results of the studies mentioned above, we established that the contents of the worksheets should focus on helping students to do the following:

- obtain a structural understanding of the mathematical entities "divergence" and "curl";

- interpret visualizations of vector fields in terms of divergence and curl using conceptual approaches: for example, a box mechanism to decide where the divergence is nonzero [67] or a paddle wheel approach to determine where the curl is nonzero [68];

- interpret, construct, and switch between field vector plots, field line diagrams, and algebraic expressions of vector fields;

- determine the divergence and curl in vector fields with $1 / r^{2}$ (spherical) and $1 / s$ (cylindrical) symmetry, which are exceptional from a mathematical point of view, but very common in electromagnetism;

- develop a conceptual understanding of Maxwell's equations in differential form, with a focus on the local character of the equations;

- enhance their understanding of the link between different strategies to determine the divergence and curl, in both mathematics and physics contexts:

- interpreting graphical representations of vector fields in terms of divergence and curl;

- calculating and interpreting mathematical expressions involving vector operators;

- applying Maxwell's equations in differential form.

We designed five sets of worksheets that can be implemented in an electrodynamics course. Although they can be used independently, we think learning will be optimal if all parts are used. The first two worksheets concern divergence and curl, respectively, from a mainly mathematical point of view. Therefore, these tutorials could also be useful for mathematics instruction. The remaining 
three tutorials link these mathematical concepts to a physics context, and concern the differential forms of Gauss's law, Faraday's law, and $\boldsymbol{\nabla} \cdot \mathbf{B}=0$ and Maxwell-Ampère's law, respectively. All sets of worksheets comprise between two and five pages.

The guided-inquiry questions on the worksheets vary in terms of format, including but not limited to questions that should be answered in a few short sentences, computational problems, questions in which students are asked to construct a sketch or diagram, questions in which students are asked to discuss student statements, hypothetical debate problems [69], and questions which ask for an opinion that requires a deep understanding of a concept or solution strategy. Typically, the questions try to provoke common student difficulties and then confront students with a situation that is not in line with the incorrect ideas. Some examples are given in Sec. II D. By using the worksheets in a tutorial format, students are encouraged to engage in discussions, and many questions include statements that may evoke such conversations.

Since the tutorials were implemented during multiple years, we were able to make extensive field notes about the use of the worksheets and the issues that students encountered when discussing the questions and their responses. After each year, we improved or reformulated some of the questions by taking experiences from tutoring and the posttest results into account. Consequently, the worksheets were developed using an iterative approach. The final version of all five sets of worksheets can be found online [70]. An instructor's guide is available upon request.

In the next section we discuss a few typical excerpts from the worksheets that show how the aforementioned focus points are brought into practice.

\section{Examples of tutorial worksheet design}

To give our students every opportunity to benefit from MERs, we carefully took into account all five design dimensions that are distinguished in the DeFT framework when developing the tutorials. The choice of the number of representations - dimension (a) — was informed both by the aims of our instruction and our students' prior attainment and dispositions. We decided to include three representations in our tutorials: field line diagrams, field vector plots, and symbolic expressions. The three representations each provide important information about the vector fields in different forms - dimension (b). Each representation conveys somewhat different information in different ways and to a different extent-dimension (c). Symbolic expressions are precise, analytical, mathematical expressions that define a vector field or represent Maxwell's equations point by point for all space. This representation allows for precise manipulation and calculation, and contains all information about the vector field in an abstract way with explicit use of coordinate systems, some of which may be unfamiliar to students. Only in situations of extremely high symmetry are global properties of the field easily gleaned from this representation. The field line diagram representation, on the other extreme, provides qualitative information about the variation of the field strength and direction in a region of space, and does so in a way many students have come to find intuitive. While field line diagrams allow for easy identification of the location of sources and sinks, they indicate strength and direction of fields only qualitatively, do not allow for precise manipulations, and are correlated with incorrect notions of divergence (see Sec. II A 2). In between these two extremes lies the field vector plot representation, which is the least familiar to our students. While not as precise or easily manipulable as the symbolic representation, relative field strength and direction are displayed on grid points in a way that provides easy access to both point-by-point and global information.

All information is distributed between these three representations, of which two are graphical and one is symbolic. Thus our MERs are partly homogeneous and partly heterogeneous. We chose not to include textual, pictorial, or audiovisual resources, since we believed that the three representations chosen afforded our students a sufficient number of productive vantage points. A general description of sequencing and switching between representations-dimensions (d) and (e) - is probably not fruitful; instead, we will illustrate them in a discussion of three excerpts from the tasks - the tutorials. We will also use these excerpts to give specific instances of the functions of MERs: to complement, constrain, and construct.

An excerpt from the first set of worksheets, the divergence tutorial, is shown in Fig. 2. Here we opted to use just the two representations that had proven fruitful for the students we interviewed: a vector field plot and a symbolic representation. Since students had spontaneously used a (different) graphical representation, we started with the vector field plot representation. We asked students to make a prediction based on this representation and only then introduced the symbolic representation which allowed them to calculate the divergence at any position. Thus dimensions (a)-(d) are easily recognized.

Dimension (e), switching, is where the function of the MERs comes to the fore and the MER approach becomes more than the sum of its parts. Here we used a deeper exploration of the symbolic expression to eventually constrain possible interpretations of the vector field plot representation. The calculation of divergence is straightforward away from the source of this field. Only at the location of the source the divergence of the field is nonzero. We chose to give the students a hint that they may need the delta distribution to describe the nonzero divergence mathematically. For many students the calculations challenge their intuitive ideas about divergence being nonzero everywhere in a $1 / s \hat{\mathbf{s}}$ cylindrical field. While the worksheet leaves it open to students to reconcile their ideas in any way they want, the tutors are primed to encourage them to 
(b) The following vector field decreases in magnitude when the distance to the origin increases.

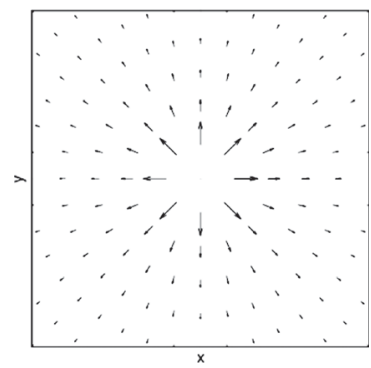

(i) At first sight, where (if anywhere) would you expect the divergence to be nonzero in the field? Why?

(ii) This field is $\mathbf{v}=\frac{c}{s} \hat{\mathbf{s}}$ with $c$ a positive constant. Calculate the divergence of the field at an arbitrary location.

(iii) What happens at the origin? (Hint: think about delta distributions)

(iv) Compare the answer in part (ii) to your expectations from part (i). If they are inconsistent, reconsider your reasoning.

(v) Can you think of a physical field that behaves like this? Give at least one example.

FIG. 2. Excerpt of a worksheet used in the divergence tutorial. In the original format, some blank space was left after every subquestion for the students to formulate their responses.

qualitatively investigate the net flow per volume in the vector field plot representation and then to apply the divergence theorem in symbolic representation. Finally, in preparation for the next tutorial students are asked to think of a physical field that behaves in this manner.

In Fig. 3, an excerpt of the Gauss's law tutorial is presented. It follows the divergence and curl tutorials, and we feel that our students are now likely to be ready to use all three representations. Our sequencing mimics that adopted by our students during the interviews. In the Gauss's law tutorial, the switching dimension (e) takes place at two levels. Students first draw their own field line diagram, remember or derive an expression for the field in cylindrical coordinates, and then use the knowledge acquired in the divergence tutorial to comment on a hypothetical student's statement (obtained from the interviews) that diverging field lines indicate nonzero divergence. Many students are sufficiently certain about where divergence is zero and nonzero that they can now use these MERs without help from the tutors to construct the understanding that the start and end of field lines show where divergence is nonzero in this representation, and that field lines spreading or coming together is irrelevant. The last two parts of the excerpt encourage students to strengthen their understanding of the relation between the vector field plot representation and the symbolic representation of vector fields.

Finally, we note that not all aspects were or must be tackled using MERs. For example, distinguishing the value
(2) A very long and thin wire with a positive linear charge distribution $\lambda$ is lying in the $z$ direction, and generates an electric field.

(a) Sketch a field line diagram of the electric field, both in the $(x, y)$-plane and the $(x, z)$-plane.

(b) How does the electric field behave in terms of the distance to the wire? Discuss what happens at the location of the wire, and at an infinite distance.

(c) Find an expression for the electric field of the charged wire as a function of the distance $s$ from the wire.

(d) Calculate the divergence of the electric field at an arbitrary point in space.

(e) Explain what happens with the divergence at the location of the wire using Gauss's law in differential form.

(f) A student states that "the divergence of an electric field is a measure of how the field lines spread out". Comment on this statement.

(g) Another possible graphical representation of the electric field in the $(x, y)$-plane is given below, with the wire in the center of the field. Show the result of your calculation of the divergence by using the box mechanism that you learned in the tutorial about the divergence.

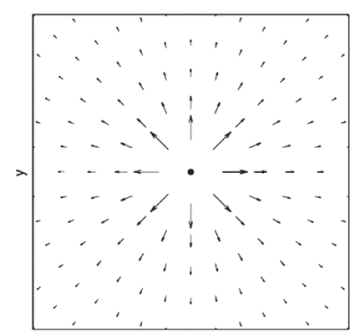

(h) When using the box mechanism, you implicitly used information about the distance dependence of the field. Explain why this is a crucial assumption.

FIG. 3. Excerpt of a worksheet used in the Gauss's law tutorial in differential form. In the original format, some blank space was left after every subquestion for the students to formulate their responses.

(4) Consider a charging capacitor as in the picture below. The plates are so close together that fringe effects can be ignored, and the finite wires carry a constant current $I$.

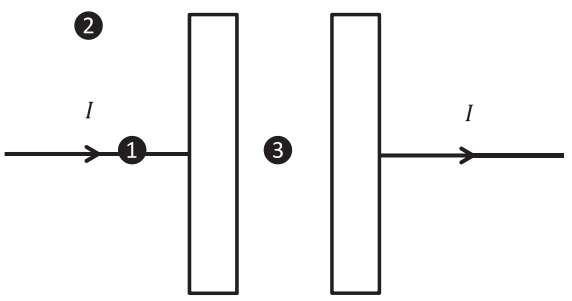

4

(a) For each of the points in the diagram above, indicate in the table below which quantities are zero and which are nonzero.

\begin{tabular}{|c|l|l|l|l|}
\hline & 1 & 2 & 3 & 4 \\
\hline $\mathbf{J}$ & & & & \\
\hline$\partial \mathbf{E} / \partial t$ & & & & \\
\hline $\mathbf{B}$ & & & & \\
\hline $\boldsymbol{\nabla} \times \mathbf{B}$ & & & & \\
\hline $\boldsymbol{\nabla} \cdot \mathbf{B}$ & & & & \\
\hline
\end{tabular}

(b) Explain how the entries in the table are consistent with Maxwell's equations.

FIG. 4. Excerpt of a worksheet used in the Maxwell-Ampère's law tutorial in differential form. 
of a field from its derivative proved a persistent difficulty and recurrent theme in our tutorials. As shown in the excerpt from the Maxwell-Ampère's law tutorial (Fig. 4), students must decide whether the magnetic field, its divergence, and its curl are zero or nonzero; thus the notion that the derivative of a function at a point generally differs from the value of the function at that point is reinforced. The students also get to consider the two possible sources of magnetic fields in a single situation: a current density and a time-varying electric field. Finally, students are asked to check their solutions for consistency with Maxwell's equations.

\section{IMPLEMENTATION OF TUTORIALS IN AN INTERMEDIATE ELECTRODYNAMICS COURSE}

The students enrolled in the second-year undergraduate electrodynamics course at KU Leuven are mainly physics and mathematics majors. All have completed an introductory electricity and magnetism course that uses the textbook by Giancoli [71] leading up to Maxwell's equations in integral form, and at least one calculus course with a chapter on vector calculus, following the textbook by Adams and Essex [72]. There are 13 weeks to a semester, and attendance is not compulsory. The course content was based on Griffiths's textbook [49]. Before the intervention, the electrodynamics course comprised one $2 \mathrm{~h}$ lecture session and one $1.5 \mathrm{~h}$ problem-solving session per week. The final exam was based mostly on exercises from the problem-solving session.

The intervention at KU Leuven was implemented in three consecutive academic years. The tutorials replaced parts of lectures and problem-solving sessions on divergence, curl, and Maxwell's equations in differential form, but also required about two additional hours being spent on these subjects [73]. In total, about $5 \mathrm{~h}$ were spent on the tutorials in a small-group setting [74].

As explained in Sec. II, we developed five sets of worksheets. At the start of the course, a pretest was given to the students and the divergence and curl tutorials were implemented. The Gauss's law, Faraday's law, and Maxwell-Ampère's law tutorials were implemented after the corresponding chapters in Griffiths's textbook [49] were discussed during the lectures. Some time after the last tutorial, the post-test was given to the students. At KU Leuven, about 30-40 students are enrolled in the electrodynamics course every year. However, since attendance usually drops during the course of a semester, only about 15-25 students per year completed the post-test.

During the tutorials, the students were greatly encouraged to work in small groups of two or three students and discuss the contents of the worksheets, using teaching approaches adopted at the University of Washington [58] and the University of Colorado Boulder [60,65,75]. Not all students attended all tutorials; there were no alternative sessions for those who did not. These discussions were supported by the teaching assistants [76], who provided guidance for students with the tutorial questions if needed and asked challenging follow-up questions. They were also responsible for answering questions about the correctness of students' responses, since no written solutions were given to the students at any point. Student groups worked at their own pace and were in charge of how much time was spent on each part of a tutorial. Post-tests were administered at the end of the tutorial, so only those who attended a particular tutorial gave responses to the corresponding post-test.

While tutorial attendance was not mandatory, the instructor in charge of the course [77] strongly encouraged the students to take part. The tutorial content was officially recognized as examinable material in the course description.

Over the years, the context of both the electrodynamics course and the intervention varied somewhat: the emphasis during the lectures was modified, the exercises during the problem-solving session were replaced, the worksheets in the tutorials were adapted, and the teaching assistants changed.

\section{ASSESSMENT OF THE INTERVENTION}

In this section we discuss the effectiveness of the intervention and explain to what extent students perceived the tutorials as being useful and enjoyable. We are convinced that evaluating learning materials is an important part of the research process that yields valuable information about optimizing learning in an intermediate physics course, and that is why we include this section. However, the numbers must be interpreted with care. First, the number of participants was small, especially preintervention. This is not uncommon for the postintroductory physics level. Second, we cannot determine with certainty whether self-selection effects play a role, but we can say that comparing results preintervention and postintervention has ecological validity. In both cases, the students were free to attend lectures and tutorials, or not; and in both cases, the examinable materials reflected the content of the tutorials. It cannot be ruled out that some students who did not attend some or all of our tutorials would have attended the more regular tutorials, or vice versa, as we have only interviewed students who attended.

In Sec. IVA, we discuss the post-test results at KU Leuven in the years the intervention took place, and compare these to data obtained after both the original instruction and the pretest results. In all cases, the pretest took place before tutorial instruction, and the post-test after all instruction on the topics on hand. We assessed the effectiveness of the tutorials at KU Leuven with regard to students' structural understanding of divergence and curl (Sec. IV A 1), their ability to interpret the vector operators in visualizations of vector fields (Sec. IVA 2), and their conceptual understanding of Maxwell's equations in differential form (Sec. IV A 3). In Sec. IV B we explain how our 
students perceived the tutorials and discuss their informal evaluation of the intervention.

\section{A. Semiquantitative assessment of the effectiveness of tutorials}

To assess the effectiveness of the tutorials we used the same pretest and post-test design we used to evaluate learning after traditional instruction. The analysis includes a comparison of the post-test results $(N=19)$ after all the original instruction discussed in our earlier work [15] with post-test results after the three years in which the tutorials were implemented $(N=60)$, where possible. In cases where we refined some of the post-test questions we limit ourselves to comparing preand post-test results.

For brevity and readability we have chosen to minimize the numerical information on statistical significance. However, it is important that we show that the pretest and post-test data for all three intervention years are equivalent and may therefore be added together. Since we use categorical data, Pearson's chi-squared test provides a suitable means of discussing whether or not the overall results were independent of each other. We found that the post-test data after intervention were statistically the same in the three years (all $p$ values found by $\chi^{2}$ testing were larger than 0.05), which allowed us to aggregate them, even though some conditions like the prior instruction, the teaching approach during the lectures, the exercises discussed during the problem-solving sessions, and the questions asked on the final exam changed in small ways over the years. We interpret our findings as confirmation that the tweaks to the lectures and tutorials were indeed "small," and that we do not need to take these into account in this discussion.

For the individual test items the error bars represent the standard error on the proportion, calculated using the expression $\sigma=\sqrt{p(1-p) / n}$. We have used the only test applicable to unpaired proportional data, a two-tailed twoproportion $z$ test, to put our conclusions on somewhat surer footing. Only for products of $n p$ or $n(1-p)$ below five is the test likely not valid; we have indicated where this may be problematic in the text below.

\section{Structural understanding of divergence and curl}

The first pretest and post-test questions were designed to gain insight into our students' concept images of divergence and curl. The concept image is defined as a unique collection of all the mental processes activated when a person encounters a certain concept [26]. The questions comprised prevalent student statements from open-ended questions and individual interviews. In three student interviews we verified that students interpreted the last version of the questions, shown in Fig. 5, as intended. They were exactly the same on the pre- and post-test. Since this
1. Indicate which of the following statements and properties are always correct when describing "the curl of a vector field".

(C1) $\square$ The curl indicates where field lines start or end.

(C2) $\square$ The curl is a measure for how much field lines bend.

(C3) $\square$ The curl points in the direction of the steepest increase.

(C4) $\square$ The curl has a direction.

(C5) $\square$ The curl is nonzero if and only if the direction of the field changes.

(C6) $\square$ The curl is a measure of the infinitesimal rotation of the field.

(C7) $\square$ The curl is a characteristic of the field, and has the same value everywhere in the field.

(C8) $\square$ The curl is represented by a vector

(C9) $\square$ The curl is represented by a scalar.

(a) Curl questions

2. Indicate which of the following statements and properties are always correct when describing "the divergence of a vector field".

(D1) $\square$ The divergence measures the source or sink of a vector field.

(D2) $\square$ The divergence is a measure for how much field lines spread apart.

(D3) $\square$ The divergence points in the direction of the steepest increase.

(D4) $\square$ The divergence has a direction

(D5) $\square$ The divergence is a measure for how much field lines bend.

(D6) $\square$ The divergence indicates where field lines start or end

(D7) $\square$ The divergence is a characteristic of the field, and has the same value everywhere in the field.

(D8) $\square$ The divergence is represented by a vector.

(D9) $\square$ The divergence is represented by a scalar.

(b) Divergence question

FIG. 5. Questions designed to assess students' correct and incorrect conceptions of curl and divergence. The correct statements are C4, C6, C8, D1, D6, and D9.

question was not on the preintervention post-test, we can only compare responses before and after the intervention.

Figure 6 shows the proportion of students who indicated that each statement in the pretest and the post-test is correct. Only students who took both tests were taken into account. The error bars represent the standard error of the proportion. It is clear that students generally improved their ideas of the concepts divergence and curl after the tutorials. Significantly fewer students indicated that $\mathrm{C} 2$ [The curl is a measure for how much field lines bend (incorrect)] and C5 [The curl is nonzero if and only if the direction of the field changes (incorrect)] were correct, while more students checked the boxes of statements D1 [The divergence measures the source or sink of the field (correct)] and D6 [The divergence indicates where field lines start or end (correct)]. However, no such improvements were seen in the most important distractor D2 [The divergence is a measure for how much field lines spread apart (incorrect)]. This may in part be due to a potentially confusing statement in the textbook [49]: "is a measure of how much the vector 


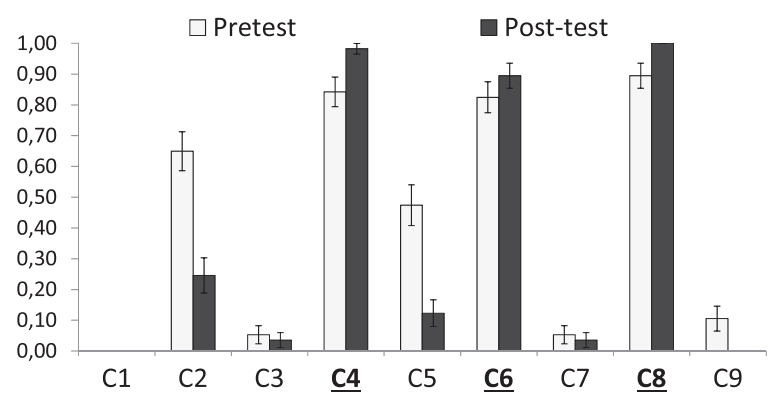

(a) Prevalence of conceptions related to curl.

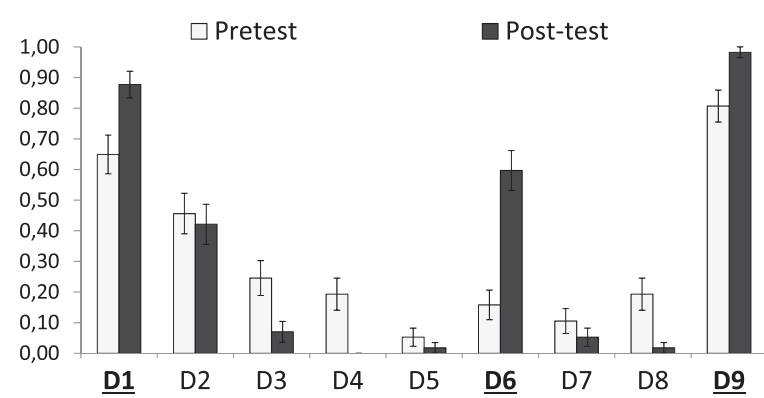

(b) Prevalence of conceptions related to divergence.

FIG. 6. The proportion of KU Leuven students $(N=57)$ who indicated that a statement presented in the questions in Fig. 5 is correct on the post-test, compared to the pretest. Only the students who took both the pre- and post-test were taken into account. Correct statements are in bold and underlined.

$\mathbf{v}$ spreads out (diverges) from the point in question." In addition, responses to statements about the vector or scalar character of both operators improved significantly (C4, $C 8 / 9$ and D4, D8/9), and students rarely confused divergence and gradient anymore (D3).

To put these results into perspective, on the pretest $21 \%$ of the students answered all questions pertaining to curl correctly, compared to $56 \%$ on the post-test. For divergence, $7 \%$ of the students answered all questions correctly on the pretest compared to $32 \%$ on the post-test. This shows that there is a significant increase in the number of correct answers, and students improved their understanding of the concepts divergence and curl. However, since we lack data about the situation after the original instruction at KU Leuven, we cannot rule out that these results are partly due to influences other than the tutorials.

\section{Interpretation of graphical representations in terms of divergence and curl}

As stated in Sec. II A 4, it is of great importance that students learn to interpret field vector plots, and in the tutorials we strongly focus on approaches that may help them to decide whether the divergence or curl is nonzero at a certain location in a visualized vector field. On both pretest and post-test we assessed our students' ability to interpret field vector plots in a mathematical context in
3. For the following vector fields, the $z$-component is zero everywhere.

(a) Indicate in the diagrams below where the divergence of the vector fields is zero, and where it is non-zero. Explain your reasoning.

(b) Indicate in the diagrams below where the curl of the vector fields is zero, and where it is non-zero. Explain your reasoning.

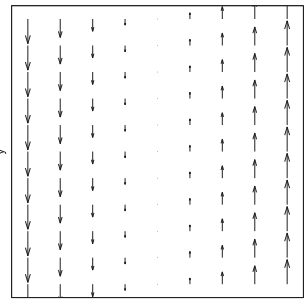

Pretest: Field (c)

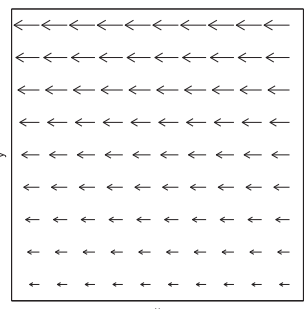

Post-test: Field (I)

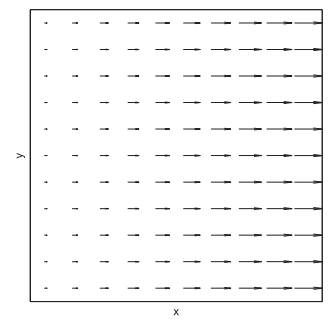

Pretest: Field (a)

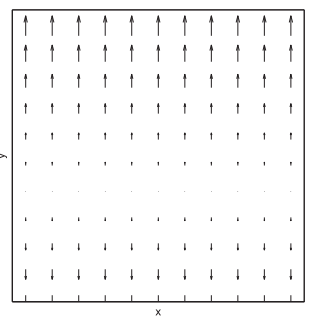

Post-test: Field (II)
FIG. 7. Question on the pre- and post-test that were given to the students in order to determine to what extent they can decide where the divergence or curl is nonzero in a field vector plot. Fields (c) and (I) are similar and yield the same solution, as is the case for fields (a) and (II).

terms of divergence and curl. The fields on the pretest are similar to those on the post-test, as can be seen in Fig. 7. Figure 8 shows the results of the pretest and post-test, for students who took part in the original instruction and for those who participated in the tutorials.

Pretest field (c) and post-test field (I) both have zero divergence and nonzero curl everywhere. Figure 8 shows that students benefited from doing the tutorials, as they responded significantly better on the post-test question after the intervention than after the original instruction. However, this trend cannot be seen when comparing results for pretest field (a) and post-test field (II), which have a nonzero divergence and zero curl everywhere. This can be explained by looking at the most prevalent error, which is stating that the divergence or curl vanishes at a location where the magnitude of the vector field is zero. Such difficulties, related to confusing the derivative with its value, were more often exhibited in field (c) and field (II) than in field (a) and field (I). Even though the worksheets specifically addressed these issues, these difficulties were not resolved for some students.

The tutorials also affected the students' approach to solving the questions in Fig. 7. In our earlier work, we discussed how we distinguish between concept-based, 


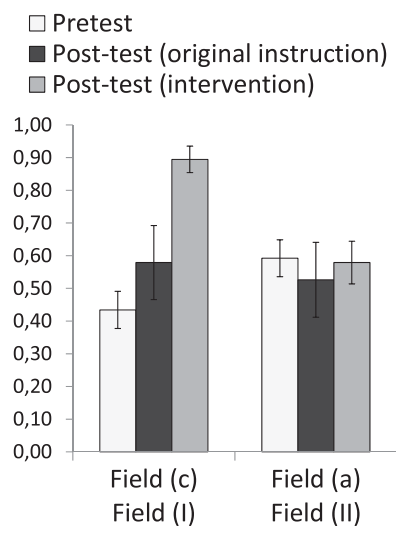

(a) Students who determined the divergence correctly.

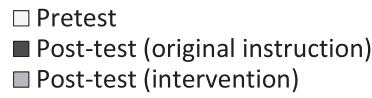

1,00

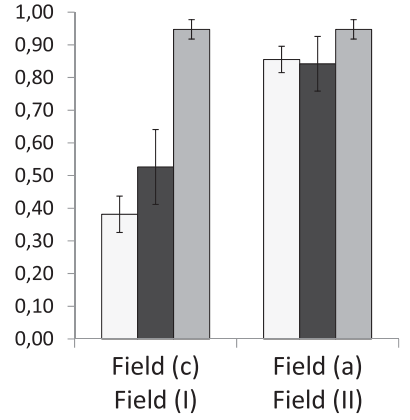

(b) Students who determined the curl correctly.

FIG. 8. The proportion of KU Leuven students who determined correctly where the divergence or curl is nonzero in a field vector plot on the pretest [field (c) and (a) in Fig. 7] versus the post-test [field (I) and (II) in Fig. 7], after the original instruction $(N=19)$ and the intervention $(N=57)$. Only the students who took both the pre- and post-test are taken into account.

formula-based, and description-based strategies when interpreting field vector plots in terms of divergence and curl [15]. A description-based strategy refers to explanations similar to "the divergence vanishes where the field is zero" or "the curl is nonzero where the field changes direction." Students who employ a formula-based strategy use the mathematical expression for the vector operators, and may even set up an equation for the vector field to calculate divergence and curl. In the worksheet, we strongly focused on concept-based strategies, like determining the net flux through a box to find out where the divergence is nonzero, or using the paddle wheel trick to decide where the curl is nonzero. This involved both demonstrating how these conceptual approaches work and giving the opportunity to apply this strategy in several exercises. Students who participated in the tutorials used such approaches in $40 \%$ (divergence) to $70 \%$ (curl) of the cases, whereas only about $20 \%$ of the students did so after the original instruction. Since such conceptual approaches correlate with a high success rate, it can explain why results after the tutorials were generally better than after the original instruction.

The post-test questions in Fig. 9 aimed to evaluate to what extent students correctly determine where the divergence or curl vanish in electric and magnetic fields. These are rather tricky questions, since from a mathematical point of view the divergence and curl behave exceptionally in vector fields that fall off as $1 / s$ (cylindrical coordinates) or $1 / r^{2}$ (spherical coordinates). While such examples were explicitly addressed in the divergence and curl tutorials, an answer can also be found by applying Maxwell's equations in differential form.

Figure 10 compares the success rate of students who took part in the original instruction with students who

4. For the following physical situations, the $z$-component of the fields is zero everywhere.

(a) Explain where the divergence of the vector fields is zero, and where it is non-zero.

(b) Explain where the curl of the vector fields is zero, and where it is non-zero.

The magnetic field of an infinite current carrying wire along the $z$-axis.

The electric field of a charged infinitely long cylinder with radius $R$.

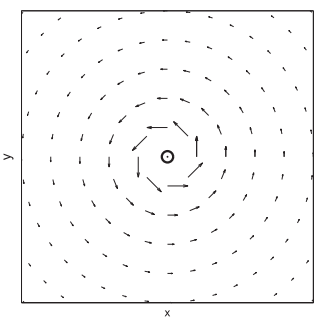

Field (III)

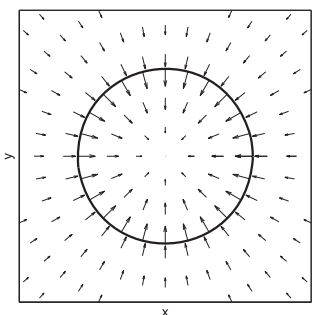

Field (IV)

FIG. 9. Post-test question to determine whether students can decide where divergence and curl are nonzero in field vector plots of electromagnetic fields.

participated in the tutorials. It seems clear that the proportion of correct responses is significantly higher for the latter cohort for field (III); the $z$ test is not reliable enough to make definitive statements for field (IV), where in any case the gains would be smaller. We also found that the students who participated in our tutorials more frequently used approaches that are generally more successful, like the conceptual approaches described above or applying Maxwell's equations in differential form, and gave descriptive answers less often. This suggests that the tutorials positively affected our students' approach and ability to determine the divergence and curl in graphical representations of electromagnetic fields.

To evaluate our students' calculational skills and their ability to construct field line diagrams, we developed the

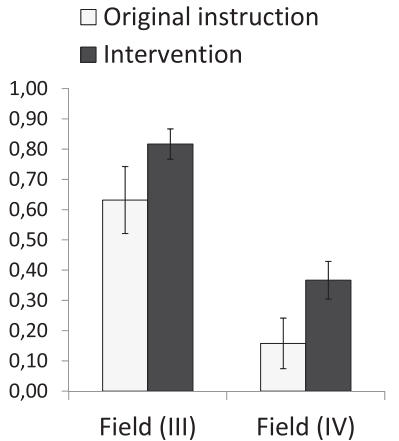

(a) Students who determined the divergence correctly.

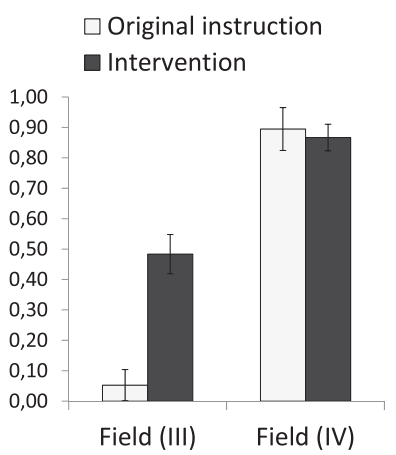

(b) Students who determined the curl correctly.
FIG. 10. The proportion of students who determined correctly where the divergence and curl are nonzero in a field vector plot of an electromagnetic field on the post-test (Fig. 9), after the original instruction $(N=19)$ and after the intervention $(N=57)$. 
5. A certain spherically symmetric vector field can be written as

$$
\mathbf{v}=-k r \hat{\mathbf{r}}
$$

where $k$ is a positive constant with appropriate units.

(a) Could this be an electrostatic field? Explain your answer.

(b) Could this be a magnetic field? Explain your answer.

(c) Sketch the field lines of this field in a region around the origin. Explain your answer.

FIG. 11. Post-test question that evaluates students' computational skills and their ability to construct a field line diagram.

post-test question in Fig. 11. The majority of the students solved parts (a) and (b) by imposing the conditions $\boldsymbol{\nabla} \times \mathbf{E}=\mathbf{0}$ and $\boldsymbol{\nabla} \cdot \mathbf{B}=0$, respectively. Over the years, the percentage of correct answers increased from $70 \%$ to $90 \%$. This confirms that students' operational conceptions were developed relatively well.

Students struggled to sketch field line diagrams of vector fields with a nonzero divergence at every location. In Fig. 12, some typical responses are shown: a correct field line diagram [Fig. 12(a)], a correct field line diagram without an indication of the direction [Fig. 12(b)], an incorrect field line diagram which resembles the electric

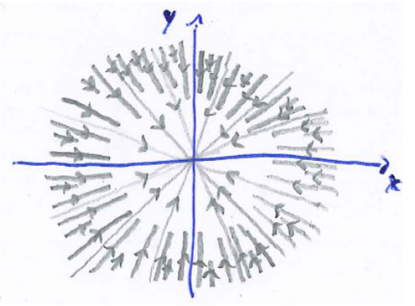

(a) Correct field line diagram.

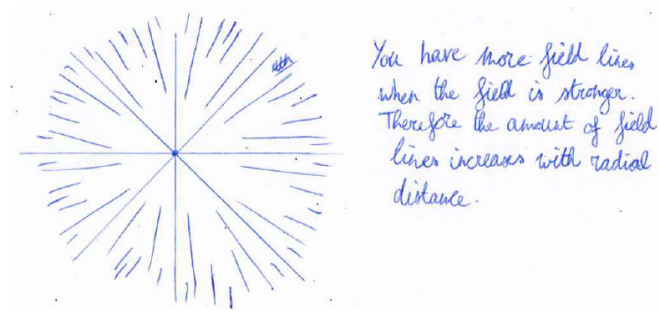

(b) Correct field line diagram without an indication of the direction.

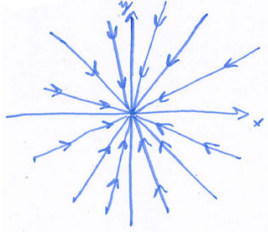

(c) Incorrect field line diagram.

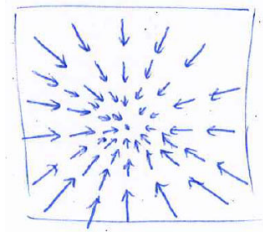

(d) Correct field vector plot.
FIG. 12. Examples of student sketches in response to post-test question 5(c) (Fig. 11). field of a point charge [Fig. 12(c)], and a correct field vector plot [Fig. 12(d)].

In the first year of implementation, $60 \%(N=15)$ of our students sketched a field line diagram and only a single student did so correctly. Two others made a sketch similar to Fig. 12(b), making a total of $20 \%$ correct answers. Most other students constructed a field line diagram similar to Fig. 12(c) or sketched (correct or incorrect) field vector plots. Because of these poor success rates, we adapted the tutorial worksheets, and this was reflected in the results. In the final two years $(N=45)$, about $25 \%$ of the students constructed a correct field line diagram and another 15\% sketched a correct diagram but did not indicate the direction of the field. However, about 30\% of the students still constructed an incorrect field line diagram, and one out of four students sketched a field vector plot. Therefore, giving students additional exercises regarding vector fields with a nonzero divergence does seem to positively influence their understanding of how to construct field line diagrams of such fields but leaves room for improvement.

\section{Understanding of Maxwell's equations in differential form}

To assess to what extent our students conceptually understood Maxwell's equations in differential form, we asked them to determine whether the divergence or curl vanishes in ten situations involving electromagnetic fields. The post-test questions describing these situations are shown in Fig. 13.

When Maxwell's equations in differential form are applied correctly in every situation of Fig. 13, one obtains the answers: $\boldsymbol{\nabla} \cdot \mathbf{E}=0$ always except for $\mathrm{E} 1 ; \boldsymbol{\nabla} \times \mathbf{E}=\mathbf{0}$ in situation E1, E3, and E5; $\boldsymbol{\nabla} \cdot \mathbf{B}=0$ always; and $\boldsymbol{\nabla} \times \mathbf{B}=\mathbf{0}$ in M4 and M5. We did not give the students a list of Maxwell's equations to avoid pointing students in a particular direction.

The fraction of students who indicated that the divergence or curl of the electromagnetic fields is zero, after the original instruction and after the tutorials respectively, is shown in Fig. 14. It is clear from Fig. 14(a) that students score significantly better after the intervention when determining the divergence in situations E2 and E3. Figure 14(b) shows that students also more often evaluate the curl in situation E2 correctly. However, even after the tutorials, only $20 \%$ of the students showed a good understanding of Gauss's law by evaluating the divergence correctly in all five situations, and 50\% seemed to conceptually understand Faraday's law as they correctly determined the curl in every situation. While the tutorials generally had a positive effect, there is room for further improvement.

Figure 14(c) shows that there was no significant difference in how often students indicated that the divergence of a magnetic field vanishes between the original instruction and the intervention, for all five situations individually. 


$$
\boldsymbol{\nabla} \cdot \mathbf{E}=0 \quad \boldsymbol{\nabla} \times \mathbf{E}=\mathbf{0}
$$

The electric field at a distance $r<R$

(E1) from the center of a uniformly charged solid sphere with radius $R$.

(E2) The electric field in a region where the magnetic field is changing with time.

(E3) The electric field at a distance $r$ from a pure electric dipole.

The electric field at the center of a

(E4) solenoid carrying a current that increases in time.

(E5)

The electric field inside a charged perfect conductor.

(a) Divergence and curl of E fields

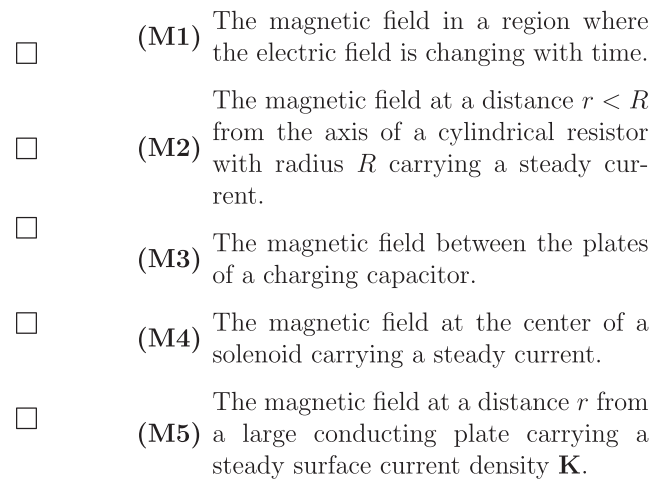

$\nabla \cdot \mathrm{B}=0 \quad \nabla \times \mathrm{B}=\mathbf{0}$

FIG. 13. Post-test question on Maxwell's equations.

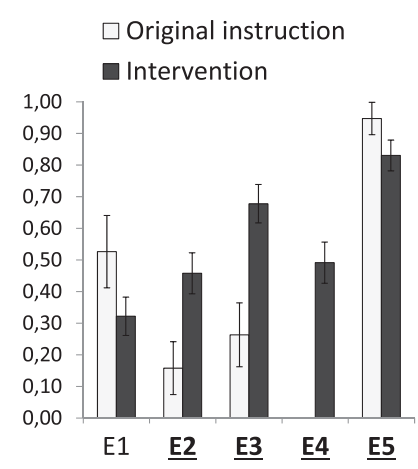

(a) $\boldsymbol{\nabla} \cdot \mathbf{E}=0$

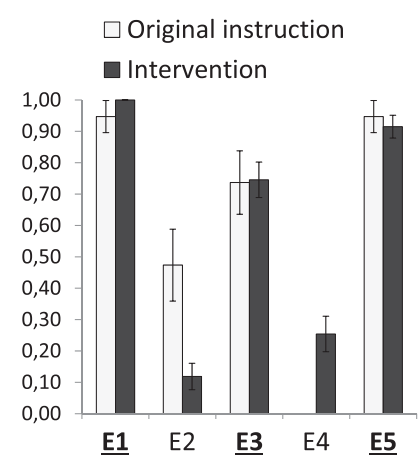

(b) $\boldsymbol{\nabla} \times \mathbf{E}=\mathbf{0}$

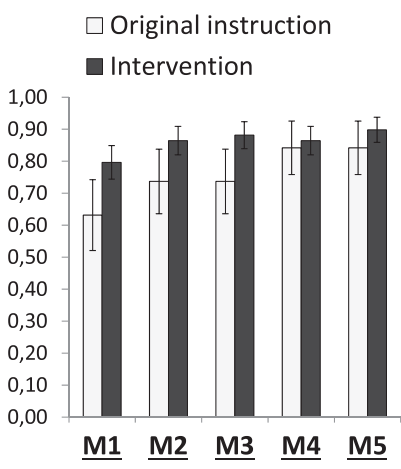

(c) $\boldsymbol{\nabla} \cdot \mathbf{B}=0$

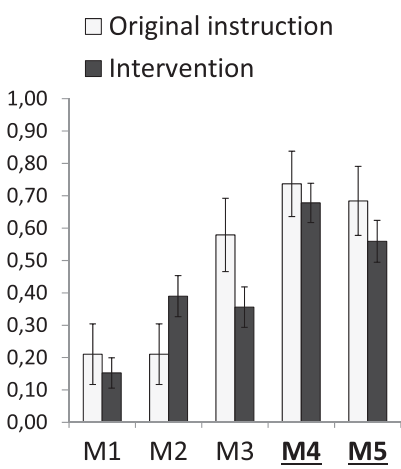

(d) $\boldsymbol{\nabla} \times \mathbf{B}=\mathbf{0}$

FIG. 14. Proportion of students who indicated the divergence or curl is zero in each situation on the post-test (Fig. 13) after the original instruction $(N=19)$ and the intervention $(N=59)$. Correct answers are in bold and underlined. We omitted the data after the original instruction for situation E4, since this situation was described ambiguously at that time.

However, while fewer than half of the students correctly checked the box of every situation on the post-test after the original instruction, more than three out of four did so after the tutorials. This suggests that considerably more students know that the divergence of the magnetic field is always zero after the intervention. No significant differences were seen when students had to decide in which of these situations the curl is zero [Fig. 14(d)]. Both after the original instruction and after the intervention, only about $20 \%$ applied MaxwellAmpère's law correctly in all five situations.

The results show that even after explicitly discussing all four of Maxwell's equations in differential form during the tutorials, students still exhibit serious difficulties with applying the laws in situations where an electromagnetic field is described. This seems to indicate that they still struggle with the conceptual meaning and local character of the equations. In Sec. V, we will further discuss the significance of these results.

\section{B. Informal evaluation}

On multiple occasions we asked students to formulate their opinion of the tutorials: in informal talk, after a oneon-one discussion about the correct post-test answers, and by inviting them to anonymously write positive and negative remarks about the intervention on a piece of paper. The most prevalent opinions from the first two years were used in eight 5-point Likert scale questions, and after the tutorial sessions in 2017 students were asked to indicate to what extent they agreed with those statements anonymously. The results are shown in Fig. 15.

In general, the students were very positive about the tutorials. They agreed that the tutorials were enjoyable and that they learned something from them. While some students told us that the tutorials were too long or that there were too many sessions, the last cohort of students generally seemed happy with the number of tutorials, and the majority was open for additional tutorials on other 


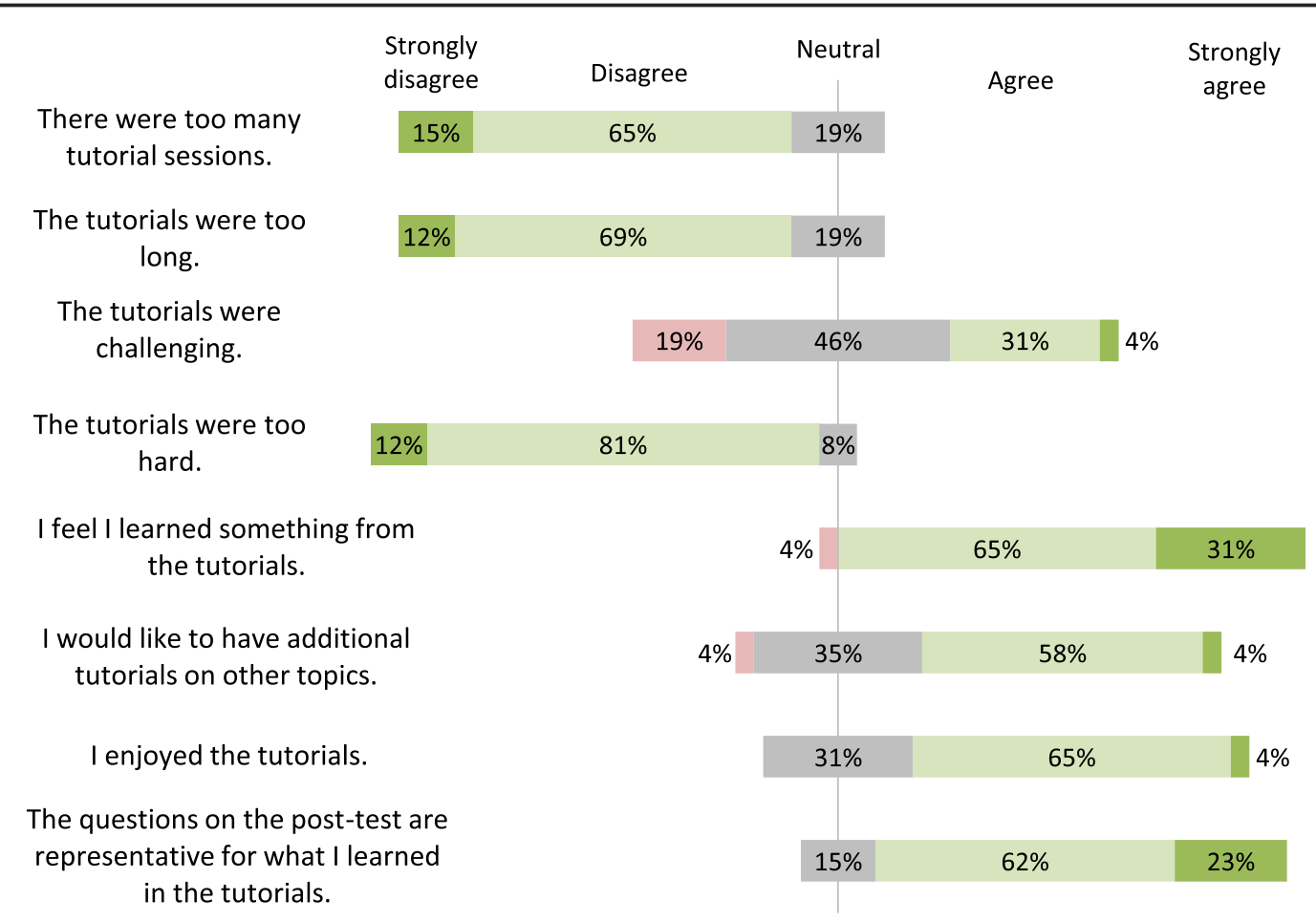

FIG. 15. Student $(N=29)$ responses to eight 5-point Likert scale questions, evaluating their experiences with the tutorials.

topics. However, for some students the tutorials were not challenging enough. While the post-test results seem to suggest that we should not make considerable changes after these comments, it is important that the teaching assistants make sure to challenge all students during the tutorials.

When asked for (other) things that students liked about the tutorials, many acknowledged that the questions on the worksheets were intuitive and forced them to think about the concepts rather than mathematical expressions. Some students explicitly stated that visualizing divergence and curl had improved their understanding of both vector calculus and Maxwell's equations in differential form. Many students also stated the tutorials helped them to understand the fundamentals of the electrodynamics course better.

The most prevalent negative comments were complaints about not getting any solution manuals for the worksheets. While we tried to discuss some important or difficult exercises on the blackboard, students felt that they should have access to all the correct answers. We believe this is unnecessary and even potentially detrimental to student engagement during the tutorials.

While many students indicated that the tutorials were useful, others stated they missed a close link to the lectures and felt there was a great contrast between the post-test questions and the type of questions that are typically asked on the final exam. We agree with the students that the integration of the tutorials in the electrodynamics course is an important issue. After each year, we discussed the results with the responsible instructor, and together we tried to improve the link between the contents on the worksheets and the subjects of the course. This is an ongoing process, and we acknowledge that further efforts are needed to improve the integration of the tutorials.

Understanding Maxwell's equations in differential form is one of the learning goals of the electrodynamics course the tutorials focus on. It is important that questions on this topic are asked on the final exam. In the first year of implementation, many students complained about the lack of relevance of these tutorials as it would not influence their final grades. Since students work toward succeeding on the final exam, this is an understandable reaction. Thankfully the responsible instructor was convinced of the importance of assessing conceptual understanding and communicated this to the students. As he adjusted some of the exam questions, students explicitly stated in the two subsequent years that they could use ideas from the tutorials during the exam. This indicates how relevant evaluation may improve students' engagement during the tutorials, and change their impression of the contents of the worksheets.

\section{DISCUSSION AND CONCLUSIONS}

Based on the difficulties identified by evaluating students' understanding of divergence and curl in mathematics and electromagnetism before and after the original instruction, conducting individual interviews, and assessing students' use of vector field representations, we designed a 
teaching-learning sequence that aims to help students with the use of vector calculus in electrodynamics. The guidedinquiry worksheets we developed aim to improve students' reasoning processes by provoking common errors based on intuitive reasoning and showing them how these incorrect responses contradict mathematical and physical principles. In addition, they demonstrate and rehearse strategies that are based on conceptual understanding and clarify the link between the divergence and curl in visualizations of vector fields, calculations, and Maxwell's equations in differential form. The worksheets were designed using a multiple representation approach, and we have discussed how the aspects that influence the effectiveness of learning with multiple representations, which are identified by the DeFT framework, are exhibited in our learning materials.

There are several ways to implement the worksheets, but for practical reasons we opted for a tutorial approach where students discuss the questions in small groups. The intervention took place in an intermediate electrodynamics course in three consecutive years (2015-2017). While no solutions were given to the students, teaching assistants were present to help students and check their responses, including for correctness.

To assess the effectiveness of the tutorials, we used the same pretest post-test design that was adopted to evaluate learning after the original instruction. The results show that our students gained insight into the mathematical concepts divergence and curl, were more successful when interpreting field vector plots in terms of divergence and curl, and generally improved their understanding of Maxwell's equations in differential form. However, the differences in post-test results between the original instruction and the tutorials were not always significant. Therefore, it is important to make some side notes when interpreting these results, which may be helpful for future instructors that plan to use (part of) our developed learning materials.

The most obvious reason for the disappointing significance of the results is the relatively small sample size. Ideally, the type of research that we discussed is conducted in large groups of students. This may be achievable for interventions in introductory courses $[60,78,79]$, but in intermediate and advanced courses, student numbers are almost always lower, which makes it difficult to justify statements on a strong statistical basis $[61,62,65,80]$. Such issues can be resolved in part by repeating the experiment over a couple of years, as we did in this study. Nevertheless, since other conditions, like the textbook used, the teaching staff, prior courses, and other factors may also change over the years, results should always be interpreted with care.

A second issue that may have affected the significance of the results is the timing of the tutorials and the post-test. Many students tend only to study in the weeks before the final exam, but the post-test was typically given to the students halfway in the semester. In addition, further alignment of tutorials and lecture may also improve the post-test results, but we do not have sufficient data to verify such a statement. Nevertheless, our students' comments indicate that a solid link between lectures and tutorials should be one of the main points of attention in the implementation process.

A third comment is related to the type of questions on the pretest and post-test. Some questions can be answered by simply checking a box, others demand a relatively short response. This allows us to easily compare pre- and post-test results and analyze how responses change over the years. However, it also gives students the opportunity to guess, and it is impossible to track such actions. Therefore, it is likely that in some cases results tended to converge to $50 \%$, making differences appear less significant than they actually are. This effect may be quite strong for the questions in Sec. IVA 3, since those were generally perceived as being difficult. Additional qualitative research might give a better idea of the size of this effect. It would be interesting, for example, to video tape students while solving questions on the worksheets, and to analyze how their reasoning processes change over time. This may also help to further improve the learning materials.

With all these provisos our results do seem to indicate that many students benefit from the tutorials we developed with a stronger focus on conceptual understanding and the ability to interpret graphical representations. This is in agreement with the literature about the use of multiple representations [38-46]. Students generally enjoyed working through the worksheets. In addition, the majority also felt they were challenged, indicated they learned something, and seemed interested to take additional tutorials. This shows that our approach engaged and motivated students. Therefore, we encourage instructors to either use the worksheets discussed in this paper or design their own learning materials based on the information we presented.

\section{ACKNOWLEDGMENTS}

We gratefully acknowledge the advice of Charles Baily with the design of the worksheets. We thank Wojciech De Roeck and the teaching assistants for making the intervention possible, and for their flexibility during the implementation of the tutorials. We would also like to thank all participating students whose cooperation made this research project possible. This publication was made possible through funding support of the KU Leuven Fund for Fair Open Access. 
[1] T. J. Bing and E. F. Redish, The cognitive blending of mathematics and physics knowledge, AIP Conf. Proc. 883, 26 (2007).

[2] T. J. Bing and E. F. Redish, Analyzing problem solving using math in physics: Epistemological framing via warrants, Phys. Rev. ST Phys. Educ. Res. 5, 020108 (2009).

[3] R. Karam, Framing the structural role of mathematics in physics lectures: A case study on electromagnetism, Phys. Rev. ST Phys. Educ. Res. 10, 010119 (2014).

[4] C. A. Manogue and T. Dray, Bridging the gap between mathematics and the physical sciences, APS Forum Educ. 13 (2004).

[5] E. F. Redish, Problem solving and the use of math in physics courses, in World View in Physics Education 2005 (2005).

[6] J. Tuminaro, Understanding students' poor performance on mathematical problem solving in physics, AIP Conf. Proc. 720, 113 (2004).

[7] J. Tuminaro and E. F. Redish, Elements of a cognitive model of physics problem solving: Epistemic games, Phys. Rev. ST Phys. Educ. Res. 3, 020101 (2007).

[8] B. R. Wilcox, M. D. Caballero, D. A. Rehn, and S. J. Pollock, Analytic framework for students' use of mathematics in upper-division physics, Phys. Rev. ST Phys. Educ. Res. 9, 020119 (2013).

[9] E. Kuo, M. M. Hull, A. Gupta, and A. Elby, How students blend conceptual and formal mathematical reasoning in solving physics problems, Sci. Educ. 97, 32 (2013).

[10] J. Larkin, J. McDermott, D. P. Simon, and H. A. Simon, Expert and Novice performance in solving physics problems, Science 208, 1335 (1980).

[11] B. L. Sherin, How students understand physics equations, Cognit. Instr. 19, 479 (2001).

[12] A. Sfard, On the dual nature of mathematical conceptions: Reflections on processes and objects as different sides of the same coin, Educ. Stud. Math. 22, 1 (1991).

[13] J. Tuminaro, A cognitive framework for analyzing and describing introductory students' use and understanding of mathematics in physics, Ph. D. thesis, University of Maryland, 2004.

[14] S. V. Chasteen, K. K. Perkins, P. D. Beale, S. J. Pollock, and C. E. Wieman, A thoughtful approach to instruction: Course transformation for the rest of us, J. Coll. Sci. Teach. 40, 70 (2011).

[15] L. Bollen, P. van Kampen, and M. De Cock, Students' difficulties with vector calculus in electrodynamics, Phys. Rev. ST Phys. Educ. Res. 11, 020129 (2015).

[16] C. Baily, L. Bollen, A. Pattie, P. van Kampen, and M. De Cock, Student thinking about the divergence and curl in mathematics and physics contexts, Proceedings of the Physics Education Research Conference 2015, College Park, MD (AIP, New York, 2015), pp. 51-54.

[17] L. Bollen, P. van Kampen, C. Baily, and M. De Cock, Qualitative investigation into students' use of divergence and curl in electromagnetism, Phys. Rev. Phys. Educ. Res. 12, 020134 (2016).

[18] L. Bollen, P. van Kampen, C. Baily, M. Kelly, and M. De Cock, Student difficulties regarding symbolic and graphical representations of vector fields, Phys. Rev. Phys. Educ. Res. 13, 020109 (2017).
[19] N. Van Labeke and S. E. Ainsworth, Applying the DeFT Framework to the Design of Multi-Representational Instructional Simulations, in AIED'01-10th International Conference on Artificial Intelligence in Education, 2001, San Antonio, Texas (IOS Press, Amsterdam, Netherlands, 2001), pp. 314-321.

[20] S. E. Ainsworth and N. Van Labeke, Using a multirepresentational design framework to develop and evaluate a dynamic simulation environment, Int. Work. Dyn. Vis. Learn. (2002).

[21] S. E. Ainsworth, DeFT: A conceptual framework for considering learning with multiple representations, Learn. Instr. 16, 183 (2006).

[22] E. Gire and E. Price, Graphical representations of vector functions in upper-division E\&M, AIP Conf. Proc. 1413, 27 (2012).

[23] E. Gire and E. Price, Arrows as anchors: An analysis of the material features of electric field vector arrows, Phys. Rev. ST Phys. Educ. Res. 10, 020112 (2014).

[24] T. Fredlund, C. Linder, J. Airey, and A. Linder, Unpacking physics representations: Towards an appreciation of disciplinary affordance, Phys. Rev. ST Phys. Educ. Res. 10, 020129 (2014).

[25] T. Dray and C. A. Manogue, The vector calculus gap: Mathematics (Does Not Equal) physics, PRIMUS 9, 21 (1999).

[26] D. Tall and S. Vinner, Concept image and concept definition in mathematics with particular reference to limits and continuity, Educ. Stud. Math. 12, 151 (1981).

[27] C. Singh and A. Maries, Core graduate courses: A missed learning opportunity?, AIP Conf. Proc. 1513, 382 (2013.

[28] B. S. Ambrose, Investigating student understanding in intermediate mechanics: Identifying the need for a tutorial approach to instruction, Am. J. Phys. 72, 453 (2004).

[29] R. E. Pepper, S. V. Chasteen, S. J. Pollock, and K. K. Perkins, Observations on student difficulties with mathematics in upper-division electricity and magnetism, Phys. Rev. ST Phys. Educ. Res. 8, 010111 (2012).

[30] C. Baily, M. Dubson, and S. J. Pollock, Researchbased course materials and assessments for upperdivision electrodynamics (E\&M II), AIP Conf. Proc. 54, 54 (2013).

[31] C. Baily and C. Astolfi, Student reasoning about the divergence of a vector field, Proceedings of the Physics Education Research Conference 2014, Minneapolis, MN (AIP, New York, 2015), pp. 31-34.

[32] D. Rosengrant, E. Etkina, and A. Van Heuvelen, An overview of recent research on multiple representations, AIP Conf. Proc. 883, 149 (2007).

[33] J. F. Wagner, C. A. Manogue, and J. R. Thompson, Representation issues: Using mathematics in upper-division physics, AIP Conf. Proc. 1413, 89 (2012).

[34] S. Ainsworth, The functions of multiple representations, Comput. Educ. 33, 131 (1999).

[35] S. Ainsworth, P. Bibby, and D. Wood, Examining the effects of different multiple representational systems in learning primary mathematics, J. Learn. Sci. 11, 25 (2002).

[36] S. Ainsworth, The educational value of multiple representations when learning complex scientific concepts, in Visualization: Theory and Practice in Science Education, 
edited by J. K. Gilbert, M. Reimer, and M. Nakhleh (Springer, Netherlands, 2008), pp. 191-208.

[37] R. Even, Factors involved in linking representations of functions, J. Math. Behav. 17, 105 (1998).

[38] J. I. Heller and F. Reif, Prescribing effective human problem-solving processes: problem description in physics, Cognit. Instr. 1, 177 (1984).

[39] A. Van Heuvelen, Learning to think like a physicist: A review of research-based instructional strategies, Am. J. Phys. 59, 891 (1991).

[40] R. J. Dufresne, W. J. Gerace, and W. J. Leonard, Solving physics problems with multiple representations, Phys. Teach. 35, 270 (1997).

[41] P. C.-H. Cheng, Unlocking conceptual learning in mathematics and science with effective representational systems, Comput. Educ. 33, 109 (1999).

[42] A. Van Heuvelen and X. Zou, Multiple representations of work energy processes, Am. J. Phys. 69, 184 (2001).

[43] D. Rosengrant, Case study: Students' use of multiple representations in problem solving, AIP Conf. Proc. 818, 49 (2006).

[44] C. J. De Leone and E. Gire, Is instructional emphasis on the use of non-mathematical representations worth the effort?, AIP Conf. Proc. 818, 45 (2006).

[45] P. B. Kohl and N. D. Finkelstein, Effect of instructional environment on physics students' representational skills, Phys. Rev. ST Phys. Educ. Res. 2, 010102 (2006).

[46] J. F. Wagner, Transfer in pieces, Cognit. Instr. 24, 1 (2006).

[47] J. Zhang and D. A. Norman, Representations in distributed cognitive tasks, Cogn. Sci. 18, 87 (1994).

[48] J. Zhang, The nature of external representations in problem solving, Cogn. Sci. 21, 179 (1997).

[49] D. J. Griffiths, Introduction to Electrodynamics, 4th ed. (Addison-Wesley, Reading, MA, 2012), p. 624.

[50] K. F. Warnick, R. H. Selfridge, and D. V. Arnold, Teaching electromagnetic field theory using differential forms, IEEE Trans. Ed. 40, 53 (1997).

[51] T. Dray and C. A. Manogue, Using differentials to bridge the vector calculus gap, Coll. Math. J. 34, 283 (2003).

[52] B. M. Notaros, Geometrical approach to vector analysis in electromagnetics education, IEEE Trans. Ed. 56, 336 (2013).

[53] H. Huang, J. Wang, C. Chen, and X. Zhang, Teaching divergence and curl in an electromagnetic field course, Int. J. Electr. Eng. Educ. 50, 351 (2013).

[54] D. F. Styer, The geometrical significance of the Laplacian, Am. J. Phys. 83, 992 (2015).

[55] B. J. Guzzetti, T. E. Snyder, G. V. Glass, and W. S. Gamas, Promoting conceptual change in science: A comparative meta-analysis of instructional interventions from reading education and science education, Read. Res. Q. 28, 116 (1993).

[56] P. S. Shaffer and L. C. McDermott, Research as a guide for curriculum development: An example from introductory electricity. Part II: Design of instructional strategies, Am. J. Phys. 60, 1003 (1992).

[57] L. C. McDermott, P. S. Shaffer, and M. L. Rosenquist (University of Washington, Physics Education Group), Physics by Inquiry: An Introduction to Physics and the Physical Sciences (John Wiley \& Sons, New York, 1996), p. 468.
[58] L. C. McDermott and P. S. Shaffer (PEG at the University of Washington), Tutorials in Introductory Physics (Prentice Hall, Upper Saddle River NJ, 2002).

[59] C. A. Manogue and K.S. Krane, Paradigms in physics: Restructuring the upper level, Phys. Today 56, 53 (2003).

[60] N. D. Finkelstein and S. J. Pollock, Replicating and understanding successful innovations: Implementing tutorials in introductory physics, Phys. Rev. ST Phys. Educ. Res. 1, 010101 (2005).

[61] C. Singh, Interactive learning tutorials on quantum mechanics, Am. J. Phys. 76, 400 (2008).

[62] S. V. Chasteen, S. J. Pollock, R. E. Pepper, and K. K. Perkins, Transforming the junior level: Outcomes from instruction and research in E\&M, Phys. Rev. ST Phys. Educ. Res. 8, 020107 (2012).

[63] D. E. Meltzer and R. K. Thornton, Resource letter ALIP-1: Active-learning instruction in physics, Am. J. Phys. 80, 478 (2012).

[64] L. Doughty, Designing, implementing and assessing guided-inquiry based tutorials in introductory physics, Ph.D. thesis, Dublin City University, 2013.

[65] C. Baily, M. Dubson, and S. J. Pollock, Developing tutorials for advanced physics students: Processes and lessons learned, in 2013 Phys. Educ. Res. Conf. Proc. (American Association of Physics Teachers, 2014) pp. 61-64.

[66] C. Singh and E. Marshman, Review of student difficulties in upper-level quantum mechanics, Phys. Rev. ST Phys. Educ. Res. 11, 020117 (2015).

[67] An approach based on the divergence theorem: When drawing a three-dimensional box in a field vector plot, one can check whether the divergence inside the box vanishes or not by determining the net flux through the box. If the net flux is nonzero, the divergence will be nonzero in at least one location inside the box.

[68] If a paddle wheel is placed at a certain location in a field vector plot, the curl in that location will be nonzero if and only if the paddle wheel rotates about its own axis.

[69] D. Hu and N.S. Rebello, Shifting college students' epistemological framing using hypothetical debate problems, Phys. Rev. ST Phys. Educ. Res. 10, 010117 (2014).

[70] See Supplemental Material at http://link.aps.org/ supplemental/10.1103/PhysRevPhysEducRes.14.020115 for all five sets of worksheets; They will also be available on http://fys.kuleuven.be/slonwf.

[71] D. C. Giancoli, Physics for Scientists and Engineers with Modern Physics, 4th ed. (Addison-Wesley, Reading, MA, 2008), p. 1328.

[72] R. A. Adams and C. Essex, Calculus: A Complete Course, 8th ed. (Pearson Education, Upper Saddle River, NJ, 2013), p. 1136.

[73] As the theory and end-of-chapter problems on divergence, curl, and Maxwell's equations were discussed separately during various sessions in the original instructional approach, this is a rough estimation.

[74] This is an average value, as students worked at their own pace. We documented that approximately $1 \mathrm{~h}$ was spent on both the divergence and curl tutorials, $1.5 \mathrm{~h}$ on the Gauss's law tutorial and $1.5 \mathrm{~h}$ in total on the Faraday's law and Maxwell-Ampère's law tutorials, excluding the pretests and post-tests. 
[75] S. V. Chasteen, R. E. Pepper, S. J. Pollock, K. K. Perkins, N. S. Rebello, P. V. Engelhardt, and C. Singh, But does it last? Sustaining a research-based curriculum in upperdivision electricity \& magnetism, AIP Conf. Proc. 1413, 139 (2012).

[76] One of the teaching assistants was directly involved in the research project. There were one or two teaching assistants for every session.

[77] The instructor in charge was not directly involved in the research nor the tutorials, but supported the project and made sure the necessary time slots were available.
[78] P. S. Shaffer and L.C. McDermott, A research-based approach to improving student understanding of the vector nature of kinematical concepts, Am. J. Phys. 73, 921 (2005).

[79] M. Kryjevskaia, A. Boudreaux, and D. Heins, Assessing the flexibility of research-based instructional strategies: Implementing tutorials in introductory physics in the lecture environment, Am. J. Phys. 82, 238 (2014).

[80] S. Goldhaber, S. Pollock, M. Dubson, P. Beale, and K. Perkins, Transforming upper-division quantum mechanics: Learning goals and assessment, AIP Conf. Proc. 1179, 145 (2009). 